Tropical Journal of Pharmaceutical Research June 2011; 10 (3): 309-316

(C) Pharmacotherapy Group, Faculty of Pharmacy, University of Benin

Benin City, 300001 Nigeria.

All rights reserved.

Available online at http://www.tjpr.org

DOI: 10.4314/tjpr.v10i3.13

Research Article

\title{
Formulation and Characterization of Glutaraldehyde Cross-Linked Chitosan Biodegradable Microspheres Loaded with Famotidine
}

\author{
Somasundaram Ramachandran, Satyamoorthy Nandhakumar and \\ Magharla Dasaratha Dhanaraju* \\ Research Lab, GIET School of Pharmacy, NH-5, Chaitanya Nagar, Rajahmundry-533 294, India
}

\begin{abstract}
Purpose: To formulate biodegradable chitosan microspheres loaded with famotidine to overcome the poor bioavailability and frequent dose administration of the drug.

Methods: Chitosan microspheres were prepared by simple emulsification technique based on glutaraldehyde crosslinking. Various process and formulation variables such as speed of emulsification, crosslinking time, drug/polymer ratio, volume of cross linking agent and volume of surfactant were optimized. The microspheres were characterized for entrapment efficiency, drug loading, in vitro drug release, surface morphology, as well as by particle size analysis, Fourier transform infrared (FTIR) spectroscopy and differential scanning calorimetry (DSC).

Results: The microspheres showed a smooth surface with a narrow particle size distribution (105 - 219 $\mu \mathrm{m})$ and an entrapment efficiency of up to $73 \%$. They exhibited controlled drug release characteristics with $85.6 \%$ of the drug released over a period of $24 \mathrm{~h}$ with an initial burst release of $26.9 \%$ in the first 2 h. Drug release followed Higuchi release kinetics. FTIR and DSC data indicate that there was no drug interaction between the drug and polymer used.
\end{abstract}

Conclusion: The chitosan microspheres could be further developed as a potential biodegradable carrier for oral controlled delivery of famotidine.

Keywords: Chitosan microspheres, Crosslinking, Controlled delivery, Famotidine, Glutaraldehyde, Biodegradable. 


\section{INTRODUCTION}

Chitosan has wide industrial applications such as lubricant, disintegrant, thickening, stabilising and suspending agent in pharmaceutical, textile and paper industries [1]. It is also a chelating agent for the removal of harmful metals in industrial and nuclear wastes, and a support for ion exchange, chelation and affinity chromatography [2]. The principal industrial source of chitin is shells of shrimp, lobster and crab.

Chitin and chitosan are distinguished by their solubility profile. Chitosan has favourable biological properties such as biodegradability, biocompatibility and non-toxicity. Chitosan was found to improve the fluidity of powder mixtures [3] and has satisfactory mucoadhesive property and good application potential [4]. Chitosan has antitumour activity, and hence chitosan microspheres bearing antineoplastic agents could be a promising carrier for cancer treatment [5]. It also holds immense promise for ophthalmic delivery [6].

The $\mathrm{pH}$-dependant solubility of chitosan is a function of the amino groups in the molecule and is a drawback for oral delivery in that chitosan microspheres formed by electrostatic interaction between a polyion and counterions become unstable in gastric fluid. This problem can be countered by irreversible chemical crosslinking. It has been demonstrated that drug diffusion from chitosan microspheres can be controlled by crosslinking with a dialdehyde such as glutaraldehyde [7].

Famotidine is a $\mathrm{H}_{2}$ receptor antagonist. It is widely prescribed in gastric and duodenal ulcers, Zollinger-Ellison syndrome and gastro esophageal reflux disease [8]. $\mathrm{H}_{2}$ receptor antagonists not only inhibit gastric secretion induced by histamine, gastrin and cholinergic stimulation, they also promote healing of duodenal ulcers [9]. $\mathrm{H}_{2}$ antihistamines, such as famotidine, have achieved clinical success with regard to the indications highlighted above. They block more than 90
$\%$ of nocturnal acid and $60-70 \%$ of daytime secretion. The relative potency of famotidine is also high when compared to other $\mathrm{H}_{2}$-antihistamines. The recommended dose for duodenal, gastric ulcers, reflux esophagitis, NSAID ulcers and ZollingerEllison syndrome is $20-40 \mathrm{mg}$ twice daily. It has been reported that the oral treatment of gastric disorders with $\mathrm{H}_{2}$ antagonist, such as famotidine and ranitidine, when used in combination with antacids, promotes local delivery of these drugs to the receptor of parietal cell wall. Local delivery also increases stomach wall receptor site bioavailability and increases efficacy of drugs to reduce acid secretion. This principle may be applied for improving systemic as well as local delivery of famotidine, thereby efficiently reducing gastric acid secretion [10].

Based on the foregoing, it would be useful to develop a formulation that deliver famotidine to sites in the stomach and thus enhance the effectiveness of the drug as well as provide sustained action. Hence, the objective of this work was to develop biodegradable microspheres of famotidine using chitosan for sustained release and site-specific delivery to the stomach.

\section{EXPERIMENTAL}

Chitosan (mol wt, ca. $40 \mathrm{KDa}$ ) was supplied by Central Institute of Fisheries and Technology, Cochin, India while famotidine was obtained as a gift from Novartis, Bombay. Sorbitan sesquioleate, glutaraldehyde (25\%, aqueous), liquid paraffin (light, viscosity $18 \mathrm{cps}$ ), and petroleum ether were obtained from Loba Chemie Pvt Ltd, Bombay. All the other chemicals used were of analytical grade.

\section{Preparation of famotidine-loaded chitosan microspheres}

Famotidine-containing chitosan microspheres were prepared at various drug: polymer ratios using a simple emulsion technique. A gel (6g) was prepared using chitosan (4\%) in $5 \%$ 
aqueous acetic acid containing $2 \% \mathrm{NaCl}$ and the drug was added to it in varying drug:polymer ratios. The mixture was dispersed in a mixture of $35 \mathrm{ml}$ liquid paraffin and $25 \mathrm{ml}$ of petroleum ether containing 0.85 $\mathrm{g}$ of sorbitan sesquioleate in a $100 \mathrm{ml}$ round bottom flask at room temperature [11]. The dispersion was stirred using stainless steel half moon-shaped paddle stirrer at $2000 \mathrm{rpm}$ for $5 \mathrm{~min}$ and then $10 \mathrm{ml}$ of glutaraldehyde saturated toluene (GST), prepared according to the method of Patel et al [12], was introduced into the flask while stirring. At the end of $30 \mathrm{~min}$, glutaraldehyde (25\%v/v, aqueous) was added and stirring continued. In preliminary trial batches, the volume of the crosslinking agent (glutaraldehyde saturated in toluene) and crosslinking time were varied from $0.5-15 \mathrm{ml}$ and $1-3 \mathrm{~h}$, respectively. The stirrer speed was also varied from 1500 $-3000 \mathrm{rpm}$. Stirring was continued for a total duration of $90 \mathrm{~min}$, at the end of which the hardened microspheres were filtered, washed several times, first with petroleum, then with acetone, $5 \%$ solution of sodium metabisulphate, and finally with water. The microspheres thus obtained were dried overnight in oven at $60{ }^{\circ} \mathrm{C}$ and stored in a desiccator.

\section{Determination of loading efficiency}

Famotidine drug content in the preparation was determined by extracting the drug from the microspheres with $0.1 \mathrm{M} \mathrm{HCl}$. In this method, the microspheres $(50 \mathrm{mg})$ were stirred in $50 \mathrm{ml}$ of $0.1 \mathrm{M} \mathrm{HCl}$ until dissolved; it was filtered through a Millipore filter and the drug content determined, after suitable dilution, at $265 \mathrm{~nm}$ by spectrophotometry (Perkin Elmer- Lambda 25). The loading efficiency (L) of the microspheres was calculated according to Eq 1 [13].

$L(\%)=\left(Q_{m} / W_{m}\right) \times 100$

where $W_{m}$ is the weight of the microspheres and $Q_{m}$ is the amount of drug present in of the microspheres.

\section{Entrapment efficiency}

Fifty milligrams of accurately weighed microspheres were crushed in a glass mortar with a pestle and suspended in $10 \mathrm{ml}$ of $0.1 \mathrm{M}$ $\mathrm{HCl}$ at $\mathrm{pH} 2$. Twenty four hours later, the solution was filtered and the filtrate analysed for drug content as described for loading efficiency. Drug entrapment (E) was calculated using Eq 2 [12].

$E(\%)=W / T \times 100$

where $\mathrm{W}$ is the actual drug content of the microspheres while $T$ is the theoretical content of the drug.

\section{Particle size analysis and characterization of surface morphology}

The size distribution of the microspheres in terms of their average diameter $\left(\mathrm{d}_{\mathrm{avg}}\right)$ was determined by optical microscopy (CosLab). Scanning electron microscopy (SEM, Hitachi S-520) was performed to characterize the surface morphology of the microspheres. was All determinations were carried out in triplicate.

\section{Fourier transform infrared (FT-IR) spectroscopy}

FT-IR spectra of chitosan, famotidine and drug-loaded microspheres were recorded on a PerkinElmer (Spectrum R-X1) instrument using $\mathrm{KBr}$ disc in the range of $4000-400 \mathrm{~cm}^{-}$ ${ }^{1}$ in order to assess structural changes that could have occurred in the drug or polymer as a result of microsphere formulation.

\section{Differential scanning calorimetry (DSC)}

As DSC is a useful tool to monitor the effect of additives on the structural behavior of a material, this technique was used to obtain information about the physicochemical interaction the microsphere material and the drug [14]. Differential scanning calorimetry (DSC) of famotidine, blank microspheres, and drug loaded microspheres was performed with a DSC facility (model 821e, Mettler 
Toledo). Measurements were performed over a temperature range of 20 to $240{ }^{\circ} \mathrm{C}$ at a heating rate of $10^{\circ} \mathrm{C} / \mathrm{min}$.

\section{In vitro release study}

In vitro release study on the microspheres $(50 \mathrm{mg}$ ) was carried out in $50 \mathrm{ml}$ of $0.1 \mathrm{M} \mathrm{HCl}$ $(\mathrm{pH} 2)$ in a bottle at $37^{\circ} \mathrm{C}$ for the first two hours to simulate gastric conditions, and then in $50 \mathrm{ml}$ of phosphate buffer ( $\mathrm{pH} \mathrm{6.8).} \mathrm{The}$ bottle was clamped in the orbital shaker (Remi) and agitated at $50 \mathrm{rpm}$. Samples $(5 \mathrm{ml})$ were collected at predetermined time intervals, for up to $24 \mathrm{~h}$, for analysis. The medium was replenished with an equal volume of the dissolution medium after each sampling. The samples were analysed with a UV spectrophotometer (Perkin Elmer Lambda 25) at $265 \mathrm{~nm}$. All determinations were in triplicate

\section{Statistical analysis}

Statistical analysis was performed with Graph Pad Instat 3 software. All the tests were run in triplicate $(n=3)$. Experimental results were expressed as mean $\pm S D$, and analyzed by one-way ANOVA for drug release data. Statistical significance was set at $p<0.05$.

\section{RESULTS}

\section{Preparation of microspheres}

Following preliminary trials, $10 \mathrm{ml}$ of glutaraldehyde saturated in toluene (GST), stirring speed of $2000 \mathrm{rpm}$ and crosslinking time of $3 \mathrm{~h}$ were adopted as processing factors for the preparation of the microspheres evaluated in this work.

\section{Drug-loading and entrapment efficiency}

Table 1 shows that drug loading efficiency increased with increasing in the proportion of the polymer relative to the drug. Variation in drug : polymer ratio from $1: 1$ to $1: 3$ produced a drug loading efficiency of $15 \pm 2$ and $23 \pm 3$ $\% \mathrm{w} / \mathrm{w}$. Further increase in polymer concentration showed slight reduction in the drug loading efficiency. (Table 1)

Similarly, the entrapment efficiency of the microspheres also increased with increase in the proportion of the polymer relative to the drug, giving entrapment values of $30 \pm 3 \%$ w/w to $75 \pm 5 \%$ w/w for drug polymer ratios of $1: 1$ and $1: 4$, respectively. However, further increase in the proportion of the polymer resulted in a decrease in entrapment efficiency.

\section{Particle size}

As Table 1 shows, the mean particle size (diameter) of the microspheres increased with increase in the proportion of polymer relative to that of drug, with $42 \%$ of the particles below $75 \mu \mathrm{m}, 35 \%$ in the range 75 $150 \mu \mathrm{m}$ and $33 \%$ in the range $150-300 \mu \mathrm{m}$. When the stirring speed was lowered to 1500 rpm, the proportion of large microspheres (150 - $300 \mu \mathrm{m}$ range) increased. On the other hand, increase in stirring speed beyond 3000 rpm lowered particle size.

\section{Scanning electron microscopy}

The SEMs of the microspheres in Fig 1 indicate that they were fairly smooth and spherical in shape with an apparently homogenous surface.

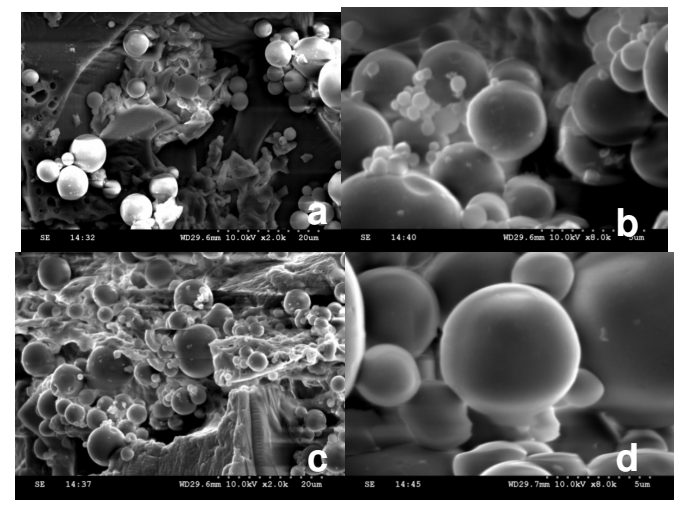

Fig 1: Scanning electron micrographs of famotidine-loaded chitosan microspheres with drug/polymer ratios of 1:3 ( $a$ and $b$ ) and 1:4 ( $c$ and d) 
Table 1: Some characteristics of famotidine microspheres

\begin{tabular}{ccccc}
\hline Batches & $\begin{array}{c}\text { Drug/Polymer } \\
\text { ratio }\end{array}$ & $\begin{array}{c}\text { Mean diameter } \\
(\boldsymbol{\mu m})\end{array}$ & $\begin{array}{c}\text { Drug loading } \\
(\mathbf{w t} \%)\end{array}$ & $\begin{array}{c}\text { Entrapment } \\
\text { efficiency }(\mathbf{w t} \%)\end{array}$ \\
\hline F1 & $1: 1$ & $105 \pm 12$ & $15 \pm 2$ & $30 \pm 3$ \\
F2 & $1: 2$ & $130 \pm 15$ & $18 \pm 3$ & $55 \pm 5$ \\
F3 & $1: 3$ & $195 \pm 14$ & $23 \pm 3$ & $73 \pm 7$ \\
F4 & $1: 4$ & $207 \pm 13$ & $21 \pm 2$ & $74 \pm 5$ \\
F5 & $1: 5$ & $219 \pm 16$ & $20 \pm 2$ & $70 \pm 7$ \\
\hline
\end{tabular}

\section{FTIR spectra}

The FTIR spectra of chitosan, famotidine and famotidine-loaded chitosan microspheres are shown in Fig 2. The FTIR spectrum of chitosan depict a characteristic absorption band at $3437 \mathrm{~cm}^{-1}$ representing the presence of a hydrogen bonded $\mathrm{OH}$ group. The amino group showed a characteristic absorption band in the region of $3400-3500 \mathrm{~cm}^{-1}$ which must have been masked by an $\mathrm{O}-\mathrm{H}$ group absorption band. The polymer also showed the characteristic bands of amide at 1654, 1608 and $1323 \mathrm{~cm}^{-1}$. The ether linkage manifested as a characteristic band at 1091 $\mathrm{cm}^{-1}$.

The spectrum of famotidine showed an $\mathrm{NH}$ group (amine) characteristic absorption band at $3505 \mathrm{~cm}^{-1}$ as well as characteristic sulfonamide bands at 3400, 1350 and 1180 $\mathrm{cm}^{-1}$. The absorption band at $1650 \mathrm{~cm}^{-1}$ represents $>\mathrm{C}=\mathrm{N}-\mathrm{H}$ group while the methylene rocking vibration of $\mathrm{C}-\mathrm{H}$ bond manifested as a characteristic band at 720 $\mathrm{cm}^{-1}$.

The spectrum of famotidine-loaded microspheres showed absorption bands at 2854, 1458 and $1367 \mathrm{~cm}^{-1}$, reflective of the glutaraldehyde-crosslinked chitosan. The bands were rather intense as a consequence of aliphatic $\mathrm{C}-\mathrm{H}$ absorption. The new sharp peak at $1610 \mathrm{~cm}^{-1}$ represents stretching vibrations of $\mathrm{C}=\mathrm{N}$ in Schiff's base formed by the reaction of glutaraldehyde and chitosan. The presence of $\mathrm{S}=\mathrm{O}$ band at $3420 \mathrm{~cm}^{-1}$, ether linkage at $1100 \mathrm{~cm}^{-1}$ and amide band at 1698 and $1500 \mathrm{~cm}^{-1}$ proves that there was no change in the functional groups present in famotidine.

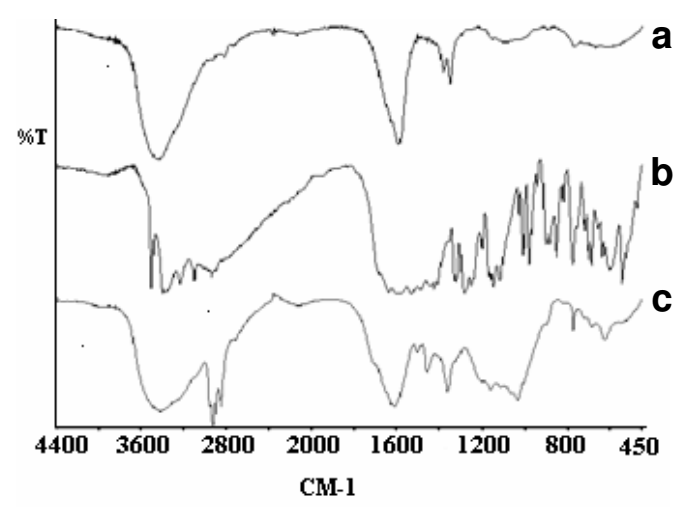

Fig 2: FTIR spectra of $(A)$ chitosan microspheres, (B) famotidine, and (C) famotidine-loaded chitosan microspheres.

\section{Differential scanning calorimetry (DSC)}

The thermograms of chitosan, famotidine drug and famotidine loaded chitosan microspheres are shown in Fig 3. The thermogram of chitosan showed a broad peak at $58^{\circ} \mathrm{C}$ which is attributed to water loss due to evaporation of absorbed water. No degradation DSC was observed for chitosan which would normally have occurred at 280 -C $[15,16]$. Famotidine thermogram showed a sharp endothermic peak at $158{ }^{\circ} \mathrm{C}$ which corresponds to its melting point which is usually in the range $157-160^{\circ} \mathrm{C}$. This peak was absent in the thermogram for the drugloaded chitosan microspheres.

\section{In vitro release}

The drug release data for the microspheres are shown in Fig 4. Cumulative drug release was 58.9, 76.0, 85.6, 84.6 and $70.3 \%$, respectively, at $1 ; 1,1 ; 2,1 ; 3,1 ; 4$ and $1: 5$ 
drug/polymer (D/P) ratio at the end of $24 \mathrm{~h}$. Te batch with a D/P ratio of $1: 3$ showed the highest initial rapid or burst release of $26.9 \%$ in $2 \mathrm{~h}$ as well as the highest cumulative drug release at the end of $24 \mathrm{~h}$.

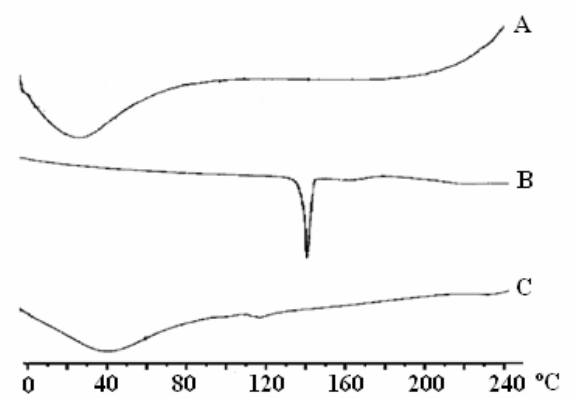

Fig 3: DSC thermograms of (a) chitosan, (b) famotidine, and (c) famotidine-loaded chitosan microspheres

On subjecting the drug release data to the Higuchi model $\left(Q=k t^{1 / 2}\right)$ in order to ascertain the drug release mechanism, a linear relationship was observed with a regression coefficient close to $1\left(r^{2}=0.993\right)$.

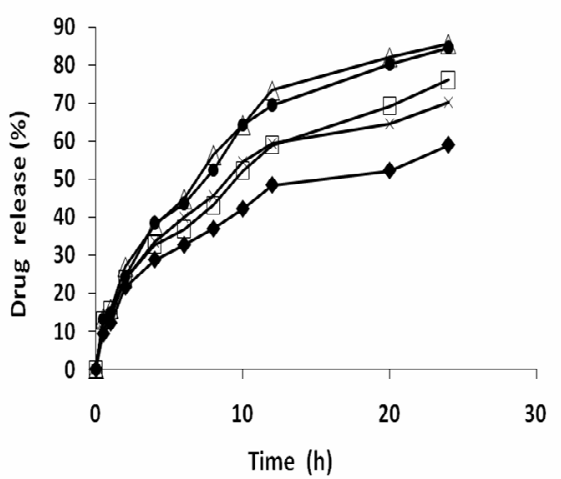

Fig 4: Famotidine release from chitosan microspheres $(\bullet=F 1(1 ; 1), \square=F 2(1 ; 2), \Delta=F 3$ $(1: 3), \bullet=F 4(1: 4), x=F 5(1: 5)$

\section{DISCUSSION}

Chitosan was selected as a polymer for the preparation of microspheres owing to its biodegradable, antiulcer, mucoadhesive properties as well as the possibility that it may offer synergism in the treatment of ulcer.

Famotidine microsphere production method was premised partly on the poor solubility of chitosan in water. Addition of acid improves the polymer's solubility as a result of the protonation of the amino groups. Its solubility is also dependent on other anions present in the solution. In the presence of acetate, lactate or glutamate, chitosan shows good solubility while phosphate, polyphosphate, sulphate and glutaraldehyde decrease its solubility [17]. For this reason, acetic acid was selected to dissolve the chitosan and glutaraldehyde was used for microsphere formation. Use of glutaraldehyde leads to the formation of a poorly soluble chitosan derivative which makes microsphere formulation become possible [11].

\section{Drug release}

When the volume of glutaraldehyde used in the microsphere preparation was increased to $15 \mathrm{ml}$, drug release decreased. This is because increase in glutaraldehyde concentration yielded highly crosslinked spheres with high density and reduced diffusion pathways. Increase in stirring speed beyond $3000 \mathrm{rpm}$ has decreased particle size to as small as $10 \mu \mathrm{m}$ thus resulting in faster drug release due to the increased surface area in contact with the dissolution medium. Increase in the crosslinking time favoured controlled release of drug from the microspheres. This is also due to the hardening of the spheres as a result of longer crosslinking time.

\section{Drug-loading and entrapment efficiency}

Several factors affect the entrapment efficiency of drugs in chitosan microspheres, e.g., nature of drug, chitosan concentration, drug polymer ratio, stirring speed, etc [18], At higher polymer concentrations, chitosan forms a highly viscous solution which is very difficult to process. 
The increase in drug-loading and entrapment efficiency with increase in the polymer content of the microspheres can be attributed to the fact that at higher polymer concentrations, chitosan viscosity leading to a less diffuse matrix structure that hinders drug escape from the microsphere core. However, excessively high plymer content would hinder homogeneous distribution of the added crosslinking agent (glutaraldehyde) leading to the formation of larger particles with reduced drug content and entrapment efficiency.

\section{Microsphere size}

Mean particle size increased with increase in polymer content of the microspheres. It seems that when polymer content increased, a more viscous internal phase manifested during the emulsification process, and was poorly dispersed in the external phase. The result is the formation of large microspheres.

\section{Compatibility issues}

FTIR and DSC results obtained indicate that there were no interactions between famotidine, chitosan and the other ingredients used in the formulation, thus suggesting that the formulations would be stable. The absence of thermogram for the drug in the drug-loaded microsphere indicates that the drug was molecularly dispersed in the polymer matrix as a solid solution.

\section{Optimum famotidine formulation}

The in vitro release profiles of F1, F2 and F3 depended mainly on drug entrapment efficiency. Although the polymer content of F3 was relatively high, it also showed a high cumulative drug release due to its high drug entrapment. Thereby the increase in drug release was in the order of $\mathrm{F} 1<\mathrm{F} 2<\mathrm{F} 3$. While drug entrapment in $\mathrm{F} 4$ was higher than in F3, it, however, showed lower drug release due to its higher polymer concentration. In F5 both lower entrapment efficiency and higher polymer concentration and these might have led to a marked reduction in drug release. Thus, F3 (drug polymer ratio, 1:3) with a rapid burst release of $26.9 \%$ in $2 \mathrm{~h}$ (due probably to a relatively high amount of drug entrapped near the surface of the microsphere) [19], and overall (sustained) release of $85.6 \%$ in $24 \mathrm{~h}$ that fitted well into Higuchi kinetic release model, can be considered, amongst all the formulations evaluated, to be the most suitable formulation in terms of reduction in frequency of administration of famotidine microsphere formulation and, thus has the potential to improve patient compliance.

\section{CONCLUSION}

The famotidine formulations developed in this study showed some potential for controlled delivery of famotidine and hence improved patient compliance. However, it would be necessary to undertake further studies, including bioavailability, in animal models with a view to determining if there is in vitro in vivo correlation.

\section{REFERENCES}

1. Upadrashta SM, Katikaneni PR, Nuessle NO. Chitosan as a tablet binder. Drug Dev Ind Pharm 1992; 18: 1701-1708.

2. Qurashi MT, Blair HS, Allen SJ. Studies on modified chitosan membranes, I. Preparation and characterization. J Appl Poly Sci 1992; 46: 255-261.

3. Sawayanagi $Y$, Nambu $N$, Nagai $T$. Directly compressed tablets containing chitin and chitosan in addition to lactose or potato starch. Chem. pharm. Bull 1982; 30: 2935-2940.

4. Miyazaki S, Nakayama A, Oda M, Takada M, Attmood D. Chitosan and sodium alginate based bioadhesive tablets for intraoral drug delivery. Biol. Pharm. Bull 1994; 17: 74547.

5. Ouchi T, Banba, Fujimoto, Hamamoto S. Synthesis and antitumor activity of chitosan carrying 5fluorouracils. Makromol chem 1989; 190: 1817-25.

6. Markey ML, Bowman L, Bergamini MVW. Chitin and chitosan; Sources, chemistry, Biochemistry, Physical Properties and Applications. Elsevier Applied Sciences, 1989; London, $p$ 713.

7. Thanoo BC, Sunny MC, JayaKrishnan A. Crosslinked chitosan microspheres: preparation and evaluation as a matrix for the controlled 
release of pharmaceuticals. J. Pharm. Pharmacol 1992; 44: 283-286.

8. Bruntan L, Lazo L, Parker KL. Goodman \& Gilman's, The Pharmacological

9. Basis of Therapeutics, $11^{\text {th }}$ edition, 2006; McGraw Hill, New York.

10. Sharma VN. Essentials of Pharmacology, $3^{\text {rd }}$ edition, CBS Publishers, New Delhi,

11. Coffin M, Parr A. US Patent 5407 687, April18, 1995.

12. Jameela SR, Jayakrishnan A. Glutaraldehyde cross linked chitosan microspheres as a long acting biodegradable drug delivery vehicle; Studies on the in vitro release of mitoxanthrone and in vivo degradation of microspheres in rat muscle. Biomaterials 1995; 16(10): 769-775.

13. Patel JK, Patel MM. Stomach Specific AntiHelicobacter Pylori Therapy: Preparation and Evaluation of Amoxicillin - Loaded Chitosan Muco adhesive Microspheres. Current Drug Delivery 2007; 4: 41-50.

14. Gladiziwa $U$, Klotz U. Pharmacokinetics \& Pharmacodynamics of $\mathrm{H}_{2}$ receptor antagonists in patients with renal insufficiency. Clin Pharmacokinetics 1993; 24: 319-332

15. 14Dhanikula $A B$, Panchagnula R. Development and characterization of biodegradable chitosan films for local delivery of paclitaxel. AAPS $J$ 2004; 6(3): Article 27, 1-5.

16. Cervera MF, Heinamaki J, Krogars K, Jorgenson AC. Solid state and mechanical properties of aqueous chitosan-Amylose starch films plasticized with polyols. AAPS Pharm Sci Tech, 2004; 5(1): Article- 15, 1-6.

17. Liao SK, Hung CC. A kinetic study of thermal degradation of chitosan/ polycaprolactam blends. Macromolecular research 2004; 12(5): 466-473.

18. Berthold A, Cremer K, Kreuter J. Preparation and characterization of chitosan microspheres as drug carrier for prednisolone sodium phosphate as model for anti-inflammatory drugs. J Controlled Release 1996; 39: 17- 25.

19. . Orienti I, Aiedeh K, Gianasi E, Bertasi V, Zecchi V. Indomethacin loaded chitosan microspheres, correlation between the erosion process \& release kinetics. J. Microencapsul, 1996; 13: 463 -472.

20. Zhon HY, Chen XG, Liu CS, Meng XH, Liu CG, Jun He, Yu LJ. Cellulose acetate/chitosan multimicrospheres preparation and ranitidine hydrochloride release in vitro. Drug delivery 2006; 13: 261-267. 